\title{
Tranexamic Acid Reduces Total Blood Loss and the Amount of Stored Preoperative Autologous Blood Donation Needed for Adolescent Idiopathic Scoliosis Patients Undergoing Posterior Spinal Fusion
}

Takahiro Hideshima ${ }^{1}$, Tsutomu Akazawa ${ }^{1}$, Masahiro Iinuma ${ }^{1}$, Yoshiaki Torii ${ }^{1}$, Jun Ueno ${ }^{1}$, Atsuhiro Yoshida ${ }^{1}$, Hisateru Niki ${ }^{1}$

1. Department of Orthopaedic Surgery, St. Marianna University School of Medicine, Kawasaki, JPN

Corresponding author: Tsutomu Akazawa, cds00350@par.odn.ne.jp

\section{Abstract}

\section{Introduction}

There are few published studies on posterior spinal fusion (PSF) for adolescent idiopathic scoliosis (AIS) that have reported that the stored amounts of autologous blood donation (ABD) needed for the procedure were estimated by taking into account total blood loss (TBL). The aim of this study was to clarify the following clinical questions: (1) Does the use of tranexamic acid (TXA) reduce the TBL during PSF for AIS? (2) What volume of ABD should be stored to avoid allogeneic blood transfusions?

\section{Methods}

This study investigated 44 female patients who underwent PSF for AIS. A total of 33 patients underwent PSF without TXA (non-TXA group) and 11 patients underwent PSF with TXA (TXA group). TBL was determined by the hemoglobin $(\mathrm{Hb})$ balance method calculated with circulating blood volume, $\mathrm{Hb}$ levels, hematocrit $(\mathrm{Ht})$ levels before and three days after surgery, and the volumes of blood transfusions, including stored ABD. For the TXA patients, TBL was used to determine the appropriate amount of stored ABD and the number of ABD collections.

\section{Results}

The amount of TBL was lower in the TXA group compared to the non-TXA group. The mean required volume of stored ABD in the TXA group was $218.2 \pm 577.3 \mathrm{~mL}$, with a required maximum volume of $699.0 \mathrm{~mL}$. The proportions of patients requiring allogeneic blood transfusion were as follows: $72.7 \%$ for those with no ABD collection, $45.5 \%$ for one ABD collection, and $0 \%$ for two ABD collections when TXA was used during surgery.

Review began 05/14/2021 Review ended 06/07/2021 Published 06/07/2021

\section{() Copyright 2021}

Hideshima et al. This is an open access article distributed under the terms of the Creative Commons Attribution License CC-BY 4.0., which permits unrestricted use, distribution, and reproduction in any medium, provided the original author and source are credited.

\section{Conclusions}

TXA reduced the TBL of patients undergoing PSF for AIS. The maximum amount of stored ABD needed was $699.0 \mathrm{~mL}$. Allogeneic blood transfusion can be avoided by storing two ABD collections when TXA is used during the surgery.

\section{Categories: Orthopedics}

Keywords: tranexamic acid, total blood loss, autologous blood donation, adolescent idiopathic scoliosis, posterior spinal fusion

\section{Introduction}

Since tranexamic acid (TXA) has been reported to reduce perioperative bleeding, several papers reported its usefulness for total hip arthroplasty (THA), total knee arthroplasty (TKA), and spinal surgery [1,2]. TXA was also reported to reduce intraoperative blood loss during posterior spinal fusion for adolescent idiopathic scoliosis (AIS) [3,4].

In addition to measurable blood loss such as intraoperative and postoperative blood loss (bleeding from a drain), Foss et al. [5]. reported that there was unmeasurable blood loss during the perioperative period. They called the unmeasurable blood loss "hidden blood loss". Intraoperative, postoperative, and hidden blood loss have been collectively defined as "total blood loss" (TBL). The methods for calculating TBL include the Gross equation, hemoglobin $(\mathrm{Hb})$ balance method, Orthopedic Surgery Transfusion Hemoglobin European Overview (OSTHEO) formula, and the Hb-dilution method [6].

Preoperative autologous blood donation (ABD) is mainly used in posterior spinal fusion (PSF) for AIS to 
avoid allogeneic blood transfusion. However, there are few published studies on PSF for AIS that have reported that the stored amount of $A B D$ needed for the procedure was estimated by taking into account the TBL. The aim of this study was to clarify the following clinical questions:

(1) Does the use of TXA reduce the TBL of PSF for AIS?

(2) What volume of ABD should be stored to avoid allogeneic blood transfusions?

We hypothesized that the use of TXA would reduce TBL and thus reduce the need for stored preoperative ABD.

\section{Materials And Methods \\ Participants}

Our institutional review board approved this retrospective study. The subjects were 52 consecutive AIS patients who underwent PSFs between August 2009 and July 2019. Eight patients were excluded. The reasons why the patients were excluded were as follows: five male patients, one patient with incomplete data, one patient with continuous injection of TXA, and one patient with anticoagulant therapy due to valvular disease. Finally, 44 patients were included in this research. The surgical procedure was pedicle screw fixation in ordinary methods. If the pedicle was small, this procedure was skipped, or we placed hooks or sublaminar bands with high molecular weight polyethylene tape. We performed inferior facetectomy (not including Ponte osteotomy) for the correction of scoliosis. After the operation, a suction drain was placed in the wound and removed on the second day after the operation. At the time of surgery, the participants' mean age was $14.5 \pm 4.4$ years, mean height was $155.1 \pm 11.2 \mathrm{~cm}$, and mean weight was $44.3 \pm 10.7 \mathrm{~kg}$. The mean $\mathrm{Hb}$ of preoperative blood samples was $13.1 \pm 1.6 \mathrm{~g} / \mathrm{dL}$ and the mean hematocrit $(\mathrm{Ht})$ was $39.4 \% \pm 4.6 \%$. The mean number of fused vertebral bodies was $9.1 \pm 5.0$ and the mean operative time was $306.6 \pm 129.7$ minutes. We divided the patients into two groups as patients with and without TXA. We started using TXA in surgery for AIS patients after July 2017, which is why some patients received TXA and others did not. There were 33 patients who underwent fusion without TXA from August 2009 to June 2017 (non-TXA group), and 11 patients who underwent fusion who received TXA from July 2017 to July 2019 (TXA group). In the TXA group, $1000 \mathrm{mg}$ TXA was intravenously administered just before surgery and every five hours thereafter until the end of surgery. TXA was not administered after surgery. The following patient factors of both groups were compared: age, height, weight, preoperative $\mathrm{Hb}$ value, preoperative $\mathrm{Ht}$ value, number of fused vertebral bodies, operative time, preoperative proximal thoracic curve (PT curve), main thoracic curve (MT curve), thoracolumbar/lumbar curve (TL/L curve), and Lenke classification [7]. The volumes of intraoperative blood loss, postoperative blood loss, and TBL (including hidden blood loss) were also compared between both groups.

\section{Calculation of TBL}

The Hb balance method [4] was used to calculate TBL. The method uses the circulating blood volume, $\mathrm{Hb}$ values before and three days after surgery, and the volume of transfused blood, which included ABD (Figure 1). 
Blood volume $(\mathrm{ml})=0.3561 \mathrm{X}(\text { Height })^{3}+0.033 \mathrm{X}$ Weight +0.1833

$\mathrm{Hb}$ transfusion $=\mathrm{Hb}$ average $\mathrm{X}$ Volume of $\mathrm{ABD}$

$\mathrm{Hb}_{\text {total loss }}(\mathrm{g})=$ Blood volume $\mathrm{X}\left(\mathrm{Hb}_{\text {pre }}-\mathrm{Hb}_{\text {post }}\right) \times 0.001+\mathrm{Hb}_{\text {transfusion }}$

$\mathrm{TBL}(\mathrm{ml})=1000 \mathrm{X} \mathrm{Hb}_{\text {total loss }} / \mathrm{Hb}$ pre

Permissible blood loss $=(\mathrm{Hb}$ pre -9$) / \mathrm{Hb}$ pre X Blood volume

Required volume of $\mathrm{ABD}=\mathrm{TBL}-$ Permissible blood loss

FIGURE 1: Calculation of total blood loss (TBL) with Hb balance method.

Blood volume $(\mathrm{ml})$ : total blood volume before surgery

Height $(\mathrm{m})$ : height before surgery

Weight (kg): weight before surgery

$\mathrm{Hb}$ transfusion $(\mathrm{g})$ : volume of hemoglobin $(\mathrm{Hb})$ in blood transfusion

ABD (ml): autologous blood donation

$\mathrm{Hb}$ average: average levels of $\mathrm{Hb}$ before $\mathrm{ABD}$

$\mathrm{Hb}$ pre $(\mathrm{g} / \mathrm{dl})$ : level of $\mathrm{Hb}$ before surgery

$\mathrm{Hb}$ post $(\mathrm{g} / \mathrm{dl})$ : levels of $\mathrm{Hb}$ three days after surgery

Permissible blood loss: blood loss keeping $\mathrm{Hb}$ levels more than $9 \mathrm{~g} / \mathrm{dl}$

Required volume of $A B D$ : appropriate volume of $A B D$

\section{Preoperative autologous blood storage}

The amount of preoperative ABD that was stored for each patient was based on guidelines for autologous blood storage [8]. For patients with a weight $\geqslant 50 \mathrm{~kg}$, the amount of blood stored at one collection was 400 $\mathrm{mL}$; and for patients with a weight $<50 \mathrm{~kg}$, the amount of blood stored at one collection was $400 \mathrm{~mL} \times$ (body weight $/ 50 \mathrm{~kg})$. For example, for a patient with a weight of $40 \mathrm{~kg}, 400 \mathrm{~mL} \times(40 / 50)=320 \mathrm{~mL}$ at one collection. $\mathrm{ABD}$ was collected and stored at 4,3 , and 2 weeks before surgery for a maximum of 3 collections. The patients were injected with $40 \mathrm{mg}$ of colloidal saccharated iron oxide (Fesin ${ }^{\circledR}$ ) and 24,000 IU of epoetin alpha at the time of collection and storage. The patients also took $100 \mathrm{mg}$ of sodium ferrous citrate tablets daily PO (Figure 2). 


\section{Cureus}

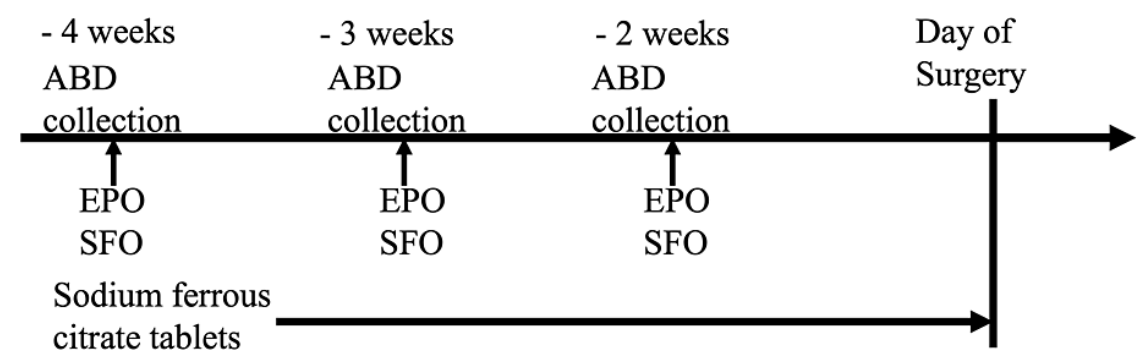

FIGURE 2: Schedule of preoperative autologous blood storage.

ABD: autologous blood donation

EPO: epoetin alfa injection $(24,000 \mathrm{U})$

SFO: saccharated ferric oxide (Fesin $₫)(40 \mathrm{mg})$

Sodium ferrous citrate tablets: $100 \mathrm{mg} /$ day from three weeks before surgery

The amount of ABD and the number of ABD collections for the TXA group

The appropriate volume of ABD collected and stored and the number of ABD collections were calculated from the TBL for the TXA group. The "Guidelines for the Use of Blood Products" from the Ministry of Health, Labor and Welfare, Japan recommended $\mathrm{Hb}$ at $7-8 \mathrm{~g} / \mathrm{dL}$ as the level indicating perioperative anemia and the need for transfusion [8]. To consider patient safety further, we used an $\mathrm{Hb}$ level of $<9 \mathrm{~g} / \mathrm{dL}$ to indicate the need for transfusion during the perioperative period. The amount of blood loss that maintained a Hb level of $\geqslant 9 \mathrm{~g} / \mathrm{dL}$ was defined as the allowable volume for blood loss (Figure 3).

FIGURE 3: The difference between total blood loss (TBL) and allowable volume for blood loss was the volume of autologous blood donation (ABD).

The proportion of patients requiring allogeneic blood transfusion was calculated for each ABD collection. In addition, the amount of $\mathrm{ABD}$ and the number of $\mathrm{ABD}$ collections needed to avoid allogeneic blood transfusion were calculated. 


\section{Cureus}

\section{Statistical methods}

Values were expressed as means \pm standard deviation. The Student T-test, Mann-Whitney U test, and Fisher exact test in EZR software (version 1.53, Saitama Medical Center, Jichi Medical University, Saitama, Japan) were used for analysis. The level of significance was set at $<5 \%$.

\section{Results}

\section{Patient demographic data}

The differences in age, height, weight, preoperative Hb level, preoperative Ht level, amount of ABD, or the number of fused vertebral bodies between both the groups were not significant. The operative time was significantly shorter for the TXA group than for the non-TXA group (Table 1). No patient sustained any major intra- or postoperative complications in either group. No adverse effects, such as venous thrombosis, headache, nausea, vomiting, or diarrhea, associated with the use of TXA were noted.

\begin{tabular}{|c|c|c|c|}
\hline & non-TXA group & TXA group & p-value \\
\hline Age, years & $14.5 \pm 4.5$ & $14.7 \pm 3.9$ & 0.73 \\
\hline Height, cm & $154.5 \pm 9.9$ & $156.6 \pm 13.8$ & 0.30 \\
\hline Weight, kg & $43.2 \pm 10.5$ & $46.3 \pm 10.0$ & 0.09 \\
\hline Preoperative $\mathrm{Hb}, \mathrm{g} / \mathrm{dl}$ & $12.9 \pm 1.6$ & $13.5 \pm 1.4$ & 0.06 \\
\hline Preoperative $\mathrm{Ht}$, \% & $39.1 \pm 4.6$ & $40.2 \pm 4.3$ & 0.16 \\
\hline Amount of ABD, ml & $1042.6 \pm 187.9$ & $1104.5 \pm 147.8$ & 0.34 \\
\hline Number of fixed vertebral bodies & $9.2 \pm 5.1$ & $8.5 \pm 4.2$ & 0.41 \\
\hline Operation time, min & $319.6 \pm 133.5$ & $267.5 \pm 75.6$ & 0.02 \\
\hline
\end{tabular}

\section{TABLE 1: Patient demographic data.}

Values were expressed as mean \pm standard deviation.

TXA: tranexamic acid, Hb: Hemoglobin, Ht: hematocrit, ABD: autologous blood donation.

\section{X-ray images}

The differences in the preoperative PT, MT, or TL/L curves between both groups were not significant. Table 2 shows that there were no significant differences in Cobb angles on X-ray images. It highlights that there was no difference in the preoperative degree of deformity between the two groups. The Lenke classifications of the patients were as follows: non-TXA group included nice patients with type I, eight with type II, four with type III, one with type IV, nine with type V, and two with type VI scoliosis. The TXA group included six patients with type I, two with type II, one with type III, and two with type V scoliosis (Table 2).

\begin{tabular}{|c|c|c|c|}
\hline & non-TXA group & TXA group & p-value \\
\hline Preoperative PT curve, degree & $27.6 \pm 25.4$ & $24.2 \pm 14.8$ & 0.42 \\
\hline Preoperative MT curve, degree & $47.1 \pm 33.4$ & $45.5 \pm 20.0$ & 0.74 \\
\hline Preoperative TL/L curve, degree & $37.1 \pm 32.4$ & $32.5 \pm 22.3$ & 0.40 \\
\hline Lenke classification (I/II/III/IV/V/VI) & $(9 / 8 / 4 / 1 / 9 / 2)$ & $(6 / 2 / 1 / 0 / 2 / 0)$ & \\
\hline
\end{tabular}

\section{TABLE 2: X-ray images.}

Values were expressed as mean \pm standard deviation

TXA: tranexamic acid, PT: proximal thoracic, MT: main thoracic, TL/L: thoracolumbar / lumbar.

\section{Blood loss}


group. The difference in the volumes of postoperative blood loss between both groups was not significant. The volume of measurable blood loss (intraoperative plus postoperative blood loss) and volume of TBL were lower in the TXA group than in the non-TXA group (Table 3). A bar graph describing the total blood loss of all surgical cases is shown in Figure 4.

\begin{tabular}{|c|c|c|c|}
\hline & non-TXA group & TXA group & p-value \\
\hline Intraoperative blood loss, ml & $1267.7 \pm 1402.3$ & $331.6 \pm 409.3$ & $<0.01$ \\
\hline Postoperative blood loss, $\mathrm{ml}$ & $765.4 \pm 577.2$ & $555.5 \pm 757.0$ & 0.07 \\
\hline Measurable blood loss, ml & $2033.1 \pm 1481.9$ & $887.1 \pm 922.1$ & $<0.01$ \\
\hline TBL, ml & $1905.3 \pm 1031.7$ & $1235.7 \pm 434.6$ & $<0.01$ \\
\hline
\end{tabular}

\section{TABLE 3: Blood loss.}

Values were expressed as mean \pm standard deviation.

TXA: tranexamic acid, TBL: total blood loss.

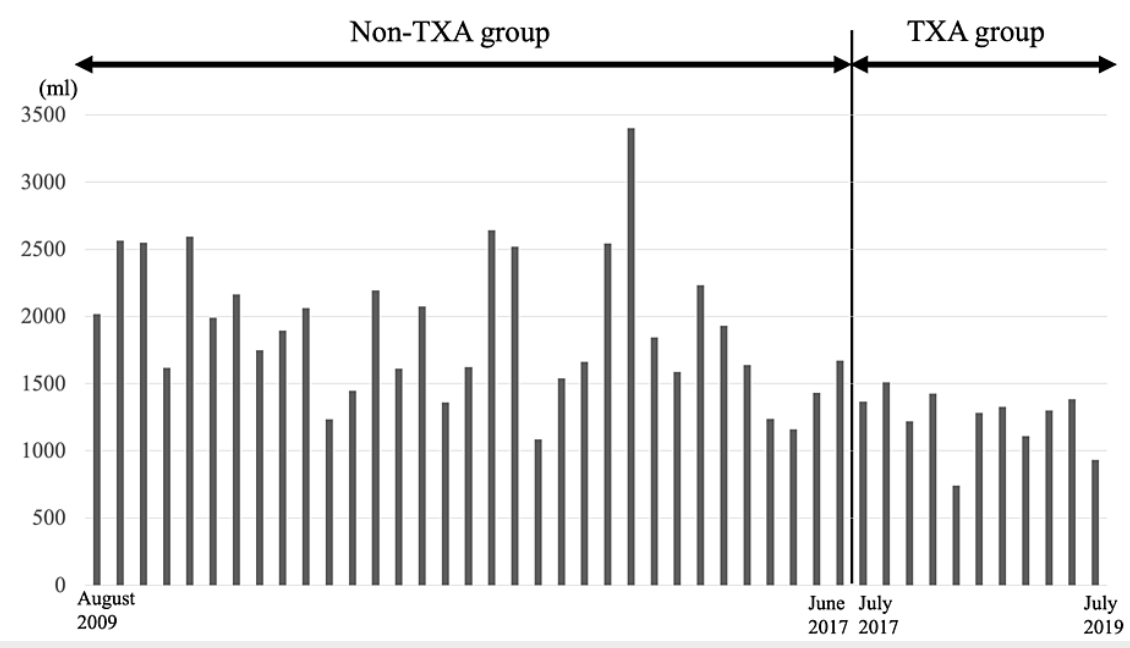

FIGURE 4: Total blood loss of all surgical cases.

The amount of $A B D$ and the number of ABD collections in the TXA group

The maximum volume of TBL in the TXA group was $1509.6 \mathrm{~mL}$, and the volume of allowable blood loss was $1017.5 \pm 259.5 \mathrm{~mL}$. The mean required volume of stored $\mathrm{ABD}$ was $218.2 \pm 577.3 \mathrm{~mL}$, and the maximum value was calculated to be $699.0 \mathrm{~mL}$. Based on these results, the proportions of patients requiring allogeneic blood transfusion were as follows: $72.7 \%$ for no ABD collection, $45.5 \%$ for one ABD collection, and $0 \%$ for two ABD collections. The required amount of $\mathrm{ABD}$ was $699.0 \mathrm{~mL}$ and the appropriate number of $\mathrm{ABD}$ collections was two.

\section{Discussion}

In our study, TXA seems to lessen ABD units that have to be stored preoperatively and thus decrease the allogenic blood transfusion rate. TXA is a drug that competitively inhibits the binding of plasmin and plasminogen to fibulin by binding to their lysine binding sites. TXA has been widely used for a long time in cardiovascular and gynecological surgery to reduce the amount of bleeding [9]. Ido et al. reported that the amounts of bleeding in total hip arthroplasty (THA) and total knee arthroplasty (TKA) procedures were reduced [1]. The amount of bleeding in spine surgery has been recently reported to be reduced when TXA is used [2], and several papers have reported that the use of TXA during posterior spinal fusion for AIS reduced intraoperative blood loss. Goobie et al. reported that the use of TXA reduced $27 \%$ of perioperative blood loss compared to a placebo group [10]. Bosch et al. reported that the transfusion rate dropped from $47 \%$ in the non-tranexamic acid cohort to $23 \%$ in the tranexamic acid cohort [11]. Eubanks reported that the use of antifibrinolytics in AIS surgery reduced blood loss and transfusion requirements [12]. Jones et al. reported 
that the use of TXA reduced the percentage of total volume lost versus no tranexamic acid in AIS patients who underwent PSF using a standardized blood loss measure [4]. Cheriyan et al. performed a meta-analysis of 11 papers reporting on 644 patients undergoing spine surgery who received TXA and found only one patient who developed an adverse effect myocardial infarction [2]. In our study, TXA reduced the amount of TBL in patients undergoing posterior spinal fusion for AIS, with no observed adverse effects. Although postoperative blood loss was not statistically different between the two groups, it might be due to the small sample size.

The reported methods of TXA administration include a single intravenous injection of 500 to $1000 \mathrm{mg}$ or an intravenous drip infusion of 500 to $2500 \mathrm{mg}$ [10,11]. In this study, intraoperative blood loss was significantly reduced in the patients receiving TXA, but the difference between the amounts of postoperative bleeding between the two groups was not significant. The effect of TXA might diminish after the procedure and did not affect postoperative blood loss. Since a comparative study based on the presence or absence of administered TXA after the surgery was not conducted, further investigation is needed. Furthermore, the question of which is more effective for AIS surgery, a single injection of TXA or continuous administration of TXA, remains unanswered $[3,10]$.

There were several reports in terms of the relationship between the use of TXA and the operative time. Sui et al. reported that the use of TXA did not affect the operative time [3]. In contrast, Berney et al. reported that the use of TXA significantly shortened the operative time [13]. In our study, TXA significantly shortened the operative time. The reduction of intraoperative blood loss might affect operative time because less blood loss made surgeons see the surgical fields better.

Foss et al. reported on the existence of hidden blood loss in addition to the measurable blood loss (intraoperative plus postoperative blood loss) and stated that TBL was defined by the measurable and hidden blood loss [5]. The Gross equation, Hb balance method, OSTHEO formula, and $\mathrm{Hb}$ dilution method have been reported to be methods for estimating the TBL [6]. In this study, the measurable blood loss in the patients receiving TXA was $736.0 \mathrm{~mL}$, whereas the estimated TBL by the $\mathrm{Hb}$ balance method was $1301.0 \mathrm{~mL}$, a difference of $565.0 \mathrm{~mL}$. In order to examine the exact amount of blood loss during the perioperative period, it would be accurate to use TBL.

In a report on AIS surgery, Ikegami et al. reported that a mean volume of $700 \mathrm{~mL}$ of $\mathrm{ABD}$ was stored, and that allogeneic blood transfusion was avoided in $96 \%$ of patients [14]. Yumite et al. reported that a stored volume of $800 \mathrm{~mL}$ of ABD avoided allogeneic blood transfusion in all their patients [15]. Our study found that the maximum volume of required amount of stored $\mathrm{ABD}$ was $699.0 \mathrm{~mL}$, and the appropriate number of ABD collection was two.

This study has limitations. It was not a randomized clinical trial. With the exception of operative times, since the differences between the demographics of the two study groups were not significant, we think that the data from the two groups of this retrospective study were sufficient for making comparisons. Because this was a retrospective study, it was difficult to adjust the number of patients and preoperative $\mathrm{Hb}$ in each group. In our study, surgeries were performed in different periods. Because TXA had been reported to reduce perioperative blood loss in several studies, we could not perform a prospective study compared between the TXA group and the non-TXA group. TXA might reduce the operative time associated with intraoperative bleeding. Despite the number of fused vertebrae not being different, we thought that the reduction of intraoperative blood loss affected the operative time because less blood loss made surgeons see the surgical fields better. The mean main thoracic curve in our study patients was approximately $45^{\circ}$, indicating that this study did not include patients with severe scoliosis. The experience and learning curve of surgeons and minor improvements in surgical techniques and hemostatic devices were not considered in our study. These factors may affect perioperative blood loss. This study only included female patients because the number of male patients was too small for analysis. We plan to continue investigating a large number of additional patients.

\section{Conclusions}

TXA reduced the TBL of patients undergoing posterior spinal fusion for AIS. The maximum amount of stored ABD needed was $699.0 \mathrm{~mL}$. Allogeneic blood transfusion can be avoided by storing two ABD collections when TXA is used during the surgery.

\section{Additional Information \\ Disclosures}

Human subjects: Consent was obtained or waived by all participants in this study. Institutional review board of St. Marianna University School of Medicine issued approval No. 4816. Animal subjects: All authors have confirmed that this study did not involve animal subjects or tissue. Conflicts of interest: In compliance with the ICMJE uniform disclosure form, all authors declare the following: Payment/services info: All authors have declared that no financial support was received from any organization for the submitted work. Financial relationships: All authors have declared that they have no financial 
relationships at present or within the previous three years with any organizations that might have an interest in the submitted work. Other relationships: All authors have declared that there are no other relationships or activities that could appear to have influenced the submitted work.

\section{References}

1. Ido K, Neo M, Asada Y, et al.: Reduction of blood loss using tranexamic acid in total knee and hip arthroplasties. Arch Orthop Trauma Surg. 2000, 120:518-20. 10.1007/s004029900132

2. Cheriyan T, Maier SP 2nd, Bianco K, et al.: Efficacy of tranexamic acid on surgical bleeding in spine surgery: a meta-analysis. Spine J. 2015, 15:752-61. 10.1016/j.spinee.2015.01.013

3. Sui WY, Ye F, Yang JL: Efficacy of tranexamic acid in reducing allogeneic blood products in adolescent idiopathic scoliosis surgery. BMC Musculoskelet Disord. 2016, 17:187. 10.1186/s12891-016-1006-y

4. Jones KE, Butler EK, Barrack T, Ledonio CT, Forte ML, Cohn CS, Polly DW Jr: Tranexamic acid reduced the percent of total blood volume lost during adolescent idiopathic scoliosis surgery. Int J Spine Surg. 2017, 11:27. 10.14444/4027

5. Foss NB, Kehlet H: Hidden blood loss after surgery for hip fracture . J Bone Joint Surg Br. 2006, 88:1053-9. 10.1302/0301-620X.88B8.17534

6. Gao FQ, Li ZJ, Zhang K, Sun W, Zhang H: Four methods for calculating blood-loss after total knee arthroplasty. Chin Med J (Engl). 2015, 128:2856-60. 10.4103/0366-6999.168041

7. Lenke LG, Betz RR, Harms J, et al.: Adolescent idiopathic scoliosis: a new classification to determine extent of spinal arthrodesis. J Bone Joint Surg Am. 2001, 83:1169-81.

8. Guidelines for autologous blood storage. Japanese Society of Autologous Blood Transfusion . (2014). http://www.jsat.jp/jsat_web/down_load/pdf/cyoketsushikijikoketsu_shishin2014_05.pdf.

9. Dunn CJ, Goa KL: Tranexamic acid: a review of its use in surgery and other indications . Drugs. 1999, 57:1005-32. 10.2165/00003495-199957060-00017

10. Goobie SM, Zurakowski D, Glotzbecker MP, et al.: Tranexamic acid is efficacious at decreasing the rate of blood loss in adolescent scoliosis surgery: a randomized placebo-controlled trial. J Bone Joint Surg Am. 2018, 100:2024-32. 10.2106/JBJS.18.00314

11. Bosch P, Kenkre TS, Soliman D, Londino JA, Novak NE: Comparison of the coagulation profile of adolescent idiopathic scoliosis patients undergoing posterior spinal fusion with and without tranexamic acid. Spine Deform. 2019, 7:910-6. 10.1016/j.jspd.2019.04.005

12. Eubanks DJ: Antifibrinolytics in major orthopaedic surgery. J Am Acad Orthop Surg. 2010, 18:132-8.

13. Berney MJ, Dawson PH, Phillips M, Lui DF, Connolly P: Eliminating the use of allogeneic blood products in adolescent idiopathic scoliosis surgery. Eur J Orthop Surg Traumatol. 2015, 25 Suppl 1:S219-23. 10.1007/s00590-015-1624-3

14. Ikegami S, Takahashi J, Kuraishi S, et al.: Efficacy of erythropoietin-beta injections during autologous blood donation before spinal deformity surgery in children and teenagers. Spine (Phila Pa 1976). 2015, 40:E1144-9. 10.1097/BRS.0000000000001108

15. Yumite Y, Ozaki T, Asaumi K, et al.: Autologous blood transfusion for scoliotic surgery. Jikoketsu Yuketsu. 2000, 13:123-6. 